Research Article

\title{
Multiobjective Optimization of Turbine Coolant Collection/Distribution Plenum Based on the Surrogate Model
}

\author{
Junsheng Chai, ${ }^{1}$ Zhenyu Wang, ${ }^{2}$ Xuanling Zhao, ${ }^{2}$ and Chunhua Wang $\mathbb{D}^{1,2}$ \\ ${ }^{1}$ AECC Shenyang Engine Research Institute, No. 1 Wanlian Road, Shenyang 110015, China \\ ${ }^{2}$ College of Energy and Power Engineering, Nanjing University of Aeronautics and Astronautics, Nanjing 210016, China
}

Correspondence should be addressed to Chunhua Wang; chunhuawang@nuaa.edu.cn

Received 29 July 2021; Revised 6 August 2021; Accepted 18 August 2021; Published 1 September 2021

Academic Editor: Jun-Wei Li

Copyright (C) 2021 Junsheng Chai et al. This is an open access article distributed under the Creative Commons Attribution License, which permits unrestricted use, distribution, and reproduction in any medium, provided the original work is properly cited.

\begin{abstract}
The turbine coolant collection/distribution chamber, as an important component of the secondary air system, undertakes the task of collecting and distributing coolant for guide vanes. To improve the outflow uniformity and reduce the flow loss, a multiobjective optimization method is developed for geometric parameters of turbine chamber. Numerical experiments were designed by Latin hypercube sampling and solved by the CFD method. Based on these data sampling, least square support vector machine (LS-SVM) was used for the surrogate model, and a kind of chaotic optimization algorithms was used for searching for the Pareto solution set. The results show that the streamline change in the optimized chamber is smoother, and the jet impingement effect of the coolant from the inlet tube was significantly weakened. At the condition that each goal has the weight of 0.5 , the optimized discharge coefficient increases by $26 \%$, and the outflow nonuniformity decreases by $79 \%$ compared with reference structure.
\end{abstract}

\section{Introduction}

With the development of aviation gas turbine engine, the compressor pressure ratio and turbine inlet gas temperature show continuous increase, and the working environment of high-temperature components also becomes worse and worse [1]. In order to ensure the safety of aeroengine components, higher requirements are put forward for the performance of the engine cooling system [2]. Turbine coolant collection/distribution chamber, as an important part of the aeroengine air system, undertakes the task of collecting and distributing coolant for guide vanes [3]. The flow loss in the turbine chamber directly affects the working efficiency of the engine. The uneven distribution of outflow will lead to insufficient or excessive coolant flow for some blades, which adversely affect the engine safety [4].

The flow loss and outflow ununiformity in turbine coolant collecting and distributing chamber are attracting more and more attentions. Zhao et al.[5] studied the flow characteristics inside the turbine chamber by using the similarity principle. It is found that, under different Reynolds num- bers, the static pressure distribution in the chamber has the contrary trend to the outflow mass distribution. Static pressure coefficient in the region facing the inlet tube directly is small, while the mass flow rate is large. The experimental results from Yao et al.[6] show that the outflow distribution of turbine chamber is ununiform. The outflow mass from the outlet hole facing the inlet tube is the largest. The total flow rate increases with the increase of the chamber height and inlet/outlet pressure ratio, but the change of these two parameters will not affect the outflow distribution law. Zhang et al.[7] investigated the influence of geometric parameters including inlet diameter, outlet hole diameter, and circumferential spacing between inlet and outlet on the outflow distribution characteristics in the turbine chamber. Their CFD and experimental results both show that at the constant pressure ratio, the geometric parameters of turbine chambers have an obvious effect on outflow nonuniformity.

The outflow distribution characteristics of turbine chamber can be affected by geometric parameters and incoming thermal parameters obviously. It is of great significance to 


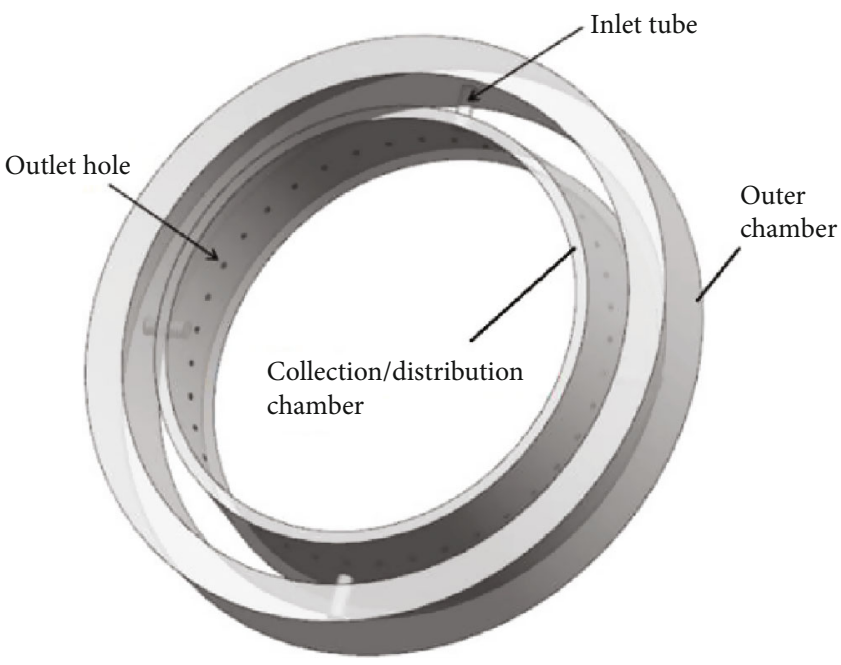

(a) 3D-view

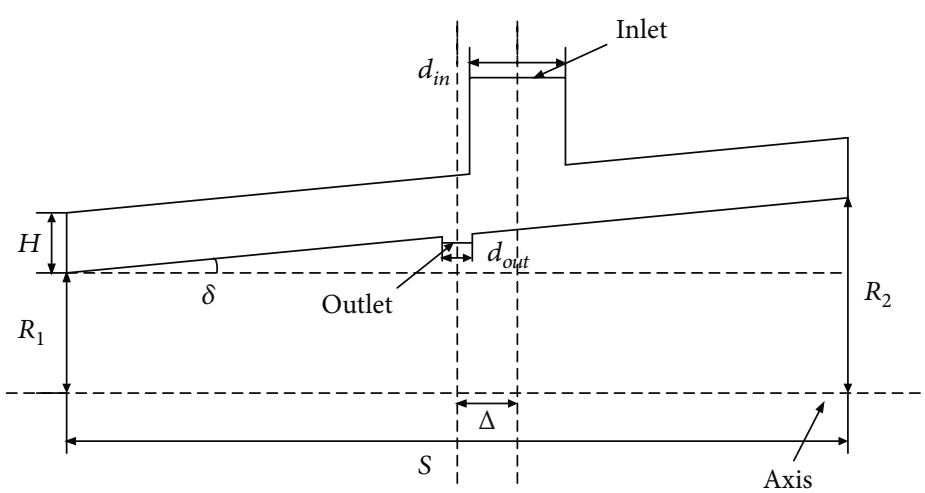

(b) Cross-section view (without outer chamber)

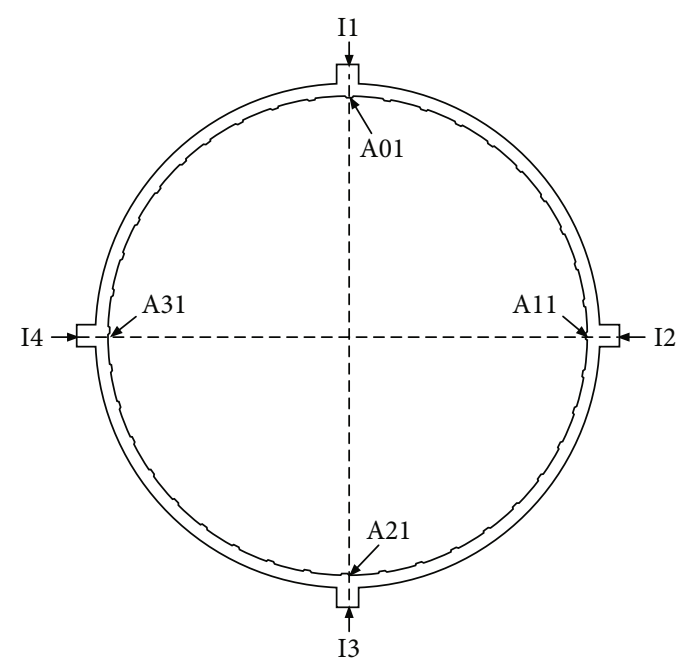

(c) Distribution of inlet tubes and outlets

FIgURE 1: Schematic of plenum chamber.

TABLE 1: Geometric parameters of reference chamber.

\begin{tabular}{lc}
\hline Geometric parameters & Value \\
\hline$H$ & $20 \mathrm{~mm}$ \\
$S$ & $262 \mathrm{~mm}$ \\
$\delta$ & $5.559^{\circ}$ \\
$R_{1}$ & $394.5 \mathrm{~mm}$ \\
$R_{2}$ & $420 \mathrm{~mm}$ \\
$d_{\text {in }}$ & $32 \mathrm{~mm}$ \\
$N_{\text {in }}$ & 4 \\
$d_{\text {out }}$ & $10 \mathrm{~mm}$ \\
$N_{\text {out }}$ & 40 \\
$\Delta$ & 0 \\
\hline
\end{tabular}

develop an optimization method for geometric parameters of turbine chamber. Lee et al. [8] performed the singleand multiobjective optimization of the fan-shaped hole on the flat plate by using surrogate model method respectively. In the single-objective optimization, maximizing the cooling efficiency was taken as the objective, and the optimized cooling effectiveness increases by $13.5 \%$ and $27.8 \%$ at the blowing ratio of 0.5 and 1.5 , respectively. In the multiobjective optimization, the cooling effectiveness and aerodynamic loss were taken into consideration simultaneously. Huang et al. [9] optimized the fan-shaped film cooling hole on the suction surface of the turbine blade. The surrogate model was established by radial basis function (RBF) neural network, and the genetic algorithm was used to search for the optimal design points. Compared with the reference structure, the area-averaged cooling effectiveness increases by $11 \sim 25 \%$ at different working conditions. For laminated cooling structures, Wang et al. [10] developed a multiobjective optimization method by combination of the RBF neural network and genetic algorithm. The optimization results show that the 

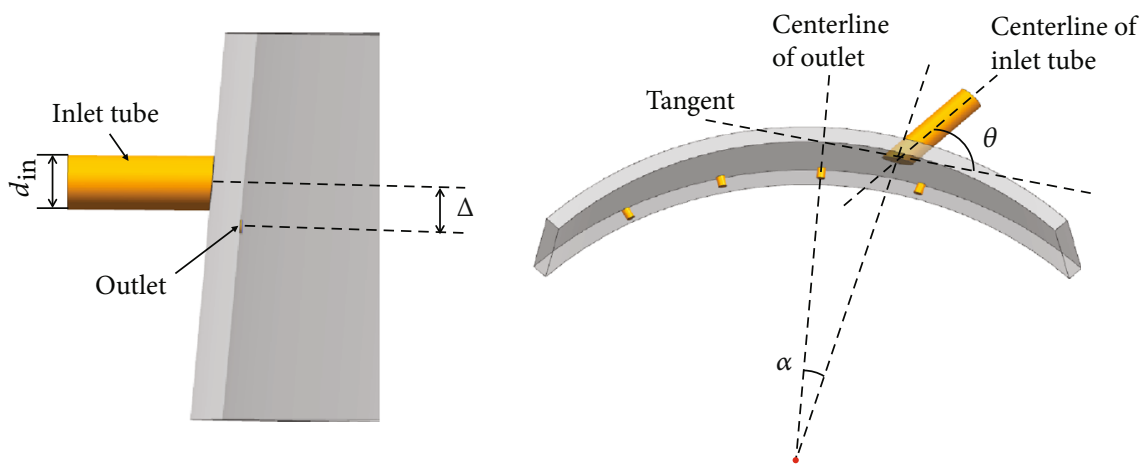

FIgURe 2: Definition of design variables.

TABLE 2: Design variables and their changing space.

\begin{tabular}{lc}
\hline Design variable & Design space \\
\hline$\Delta$ & $040 \mathrm{~mm}$ \\
$\theta$ & $2090^{\circ}$ \\
$d_{\text {in }}$ & $3046 \mathrm{~mm}$ \\
$\alpha$ & $08^{\circ}$ \\
\hline
\end{tabular}

cooling effectiveness and aerodynamic loss were improved simultaneously by optimization.

To reduce the flow loss and improve the outflow uniformity of turbine chamber, least square-support vector machine (LS-SVM) and chaotic optimization algorithm were coupled to optimize the geometric parameters. In the paper, the optimization problem for turbine chamber is introduced firstly, and then the CFD model is established and tested by the experimental data; finally, the multiobjective optimization is performed, and the flow characteristics in turbine chamber before and after optimization were compared and analyzed in detail. As far as authors know, the similar optimization research for turbine coolant chamber has not been open published yet.

\section{Computational Domain}

Figure 1 shows the reference structure of the turbine coolant collecting/distribution chamber. The coolant from the outer annular chamber enters the coolant collecting/distribution chamber through four air inlet tubes (numbered I1 I4) and then leaves the chamber from 40 outlet holes (numbered A01 A40). The height of the outer chamber is $100 \mathrm{~mm}$. The height of the annular cavity is $H$, and the axial length is $S$. The inner radius at the front end and tail end is $R_{1}$ and $R_{2}$, respectively. The diameter of the inlet hole and the outlet hole is $d_{\text {in }}$ and $d_{\text {out }}$, respectively. In the axial direction, the relative front distance between the outlet hole and the inlet tube is $\Delta$. The outlet hole with the number of A01 is facing the inlet tube I1 directly, and the number of the other outlet hole facing the inlet tubes I2, I3, and I4 is A11, A21, and A31, respectively. Table 1 shows the value of geometric parameters for the reference structure.

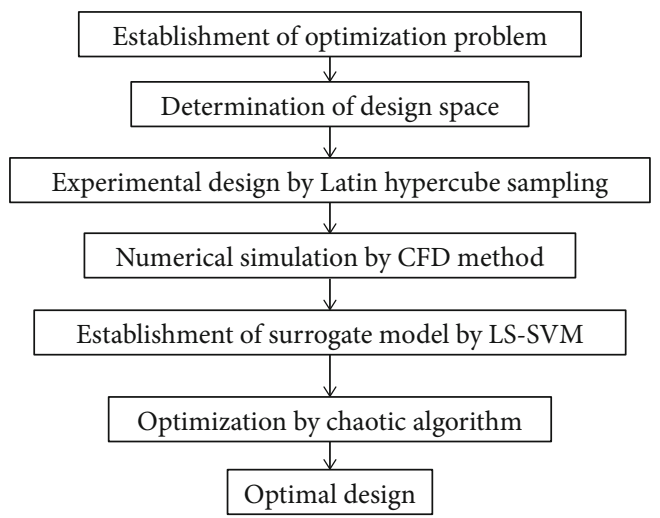

FIgURE 3: Flow chart of optimization.

Based on the reference structure, four geometric parameters are selected for design parameters. They are inlet tube diameter $\left(d_{\text {in }}\right)$, axial space between inlet and outlet $(\triangle)$, inclination angle of inlet tube $(\theta)$, and included angle between inlet and outlet $(\alpha)$. The definition of these four design parameters is shown in Figure 2. The changing interval of these parameters is shown in Table 2.

Outflow nonuniformity, $\sigma$, is used to evaluate the outflow uniformity from outlet holes and can be expressed by

$$
\begin{gathered}
\sigma=\frac{\sqrt{\sum_{i=1}^{N_{\text {out }}\left(m_{i}-\bar{m}\right)^{2} / N_{\text {out }}}}}{\bar{m}}, \\
\bar{m}=\frac{\sum_{i=1}^{N_{\text {out }}} m_{i}}{N_{\text {out }}},
\end{gathered}
$$

where $m$ is the mass flow rate, $i$ is the number of outlet, and the superscript "_" denotes the averaged value.

Discharge coefficient, $C_{d}$, is used for evaluating the flow loss and can be expressed by

$$
C_{d}=\frac{\sum_{i=1}^{N_{\text {out }}} m_{i}}{S_{a} \sqrt{2 \rho\left(P_{\text {in }}^{*}-P_{\text {out }}\right)}}
$$

where $S_{a}$ is the cross-section area of all the outlets, $P_{\text {in }}^{*}$ 


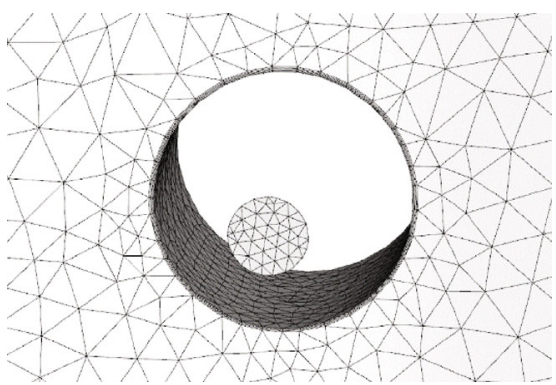

(a) Grid details

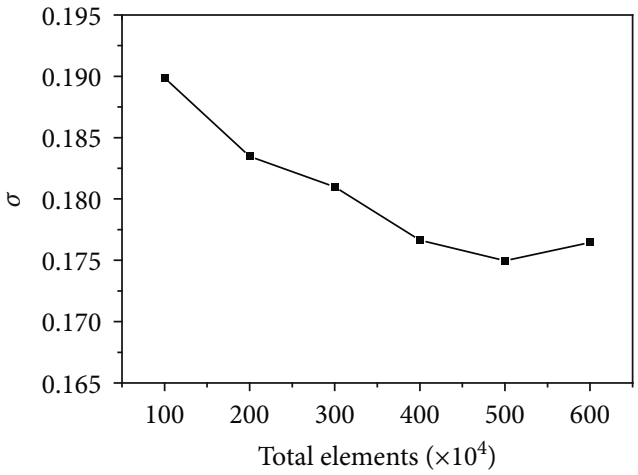

(b) Grid independent results

FIGURE 4: Grids used in the present study.
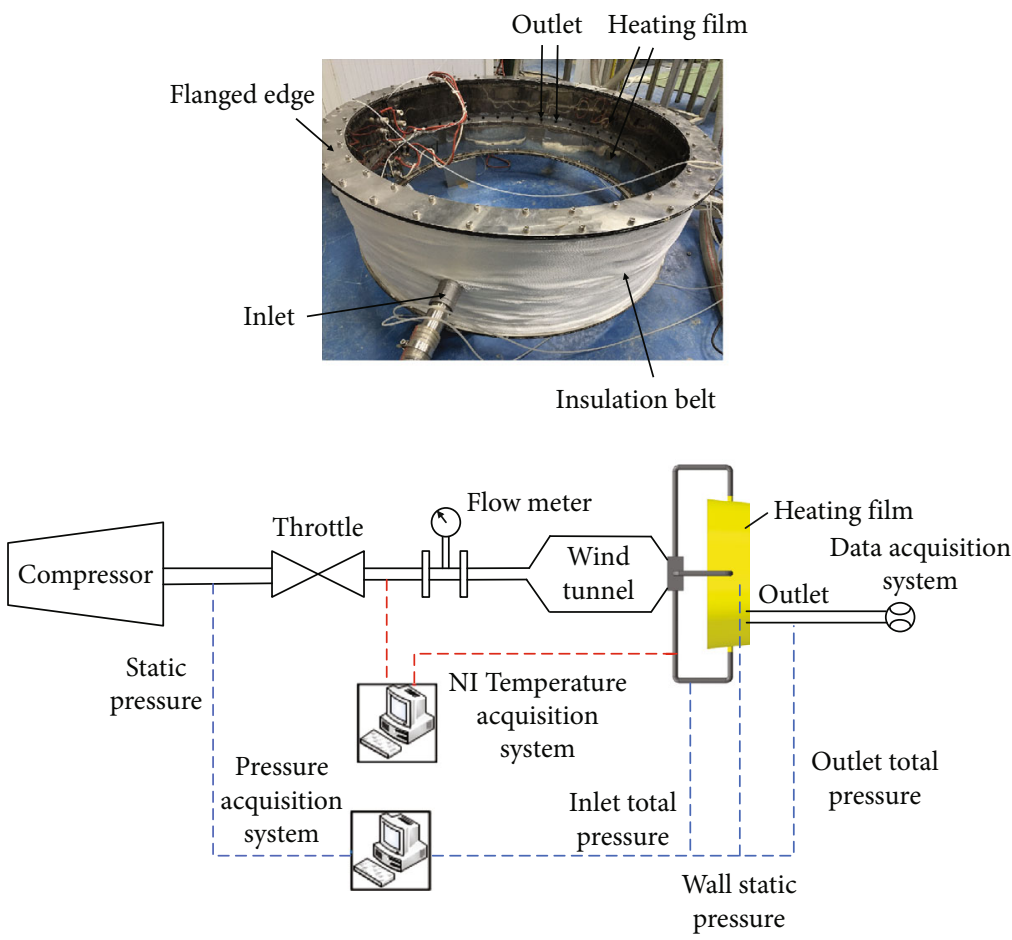

Figure 5: Experimental system.

and $P_{\text {out }}$ are the inlet total pressure and outlet static pressure respectively, and $\rho$ is the fluid density.

\section{Optimization Method}

3.1. Basic Steps. To improve the discharge coefficient and reduce the nonuniformity of outflow, inlet tube diameter $\left(d_{\text {in }}\right)$, axial space between inlet and outlet $(\triangle)$, inclination angle of inlet tube $(\theta)$, and included angle between inlet and outlet $(\alpha)$ were selected as design parameters. The mathematical description of this optimization problem is as follows.

$$
\min F\left(\Delta, \alpha, d_{\mathrm{in}}, \theta\right)=\gamma \times \sigma+(1-\gamma) \times \frac{1}{100 C_{\mathrm{d}}},
$$

$$
\text { s.t. }\left\{\begin{array}{l}
\Delta \in[0,40] \mathrm{mm}, \\
\theta \in[20,90]^{\circ}, \\
d_{i \mathrm{n}} \in[30,46] \mathrm{mm}, \\
\alpha \in[0,8]^{\circ},
\end{array}\right.
$$

where the index weight is $\gamma \in[0,1]$. As $\gamma$ is equal to 0 or 1 , the multigoal optimization converts into the single-goal optimization.

As shown in Figure 3, the optimization process can be divided into the following three steps:

Step 1. Design the numerical experiments by Latin hypercube sampling (LHS) method and establish the training and testing data sampling by the CFD solution method. 


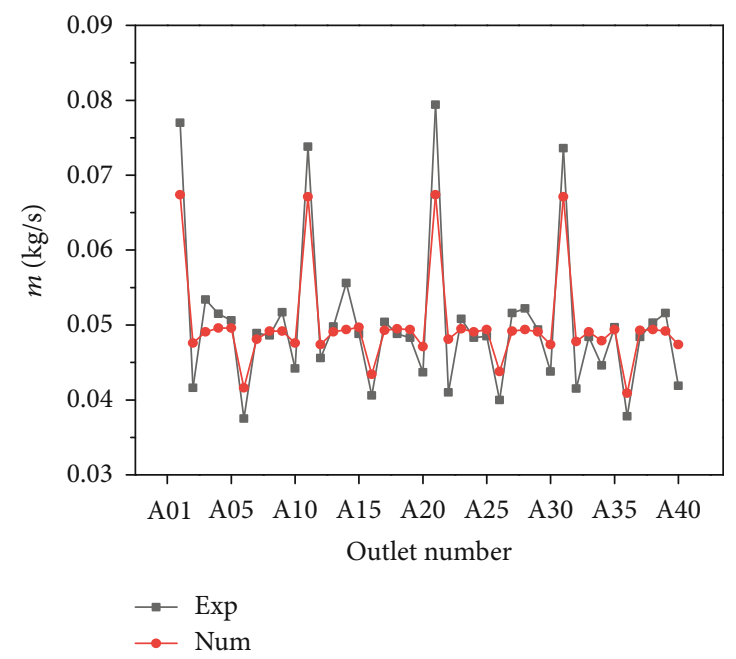

Figure 6: Numerical results vs. experimental results.

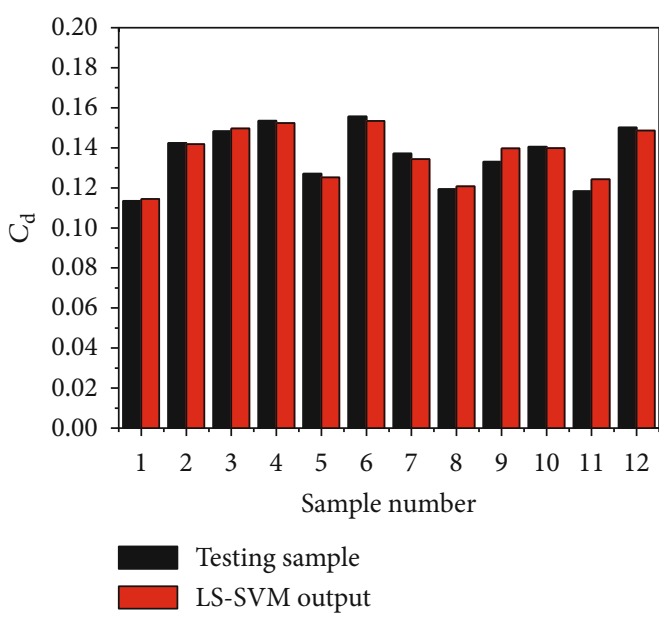

(a) Discharge coefficient

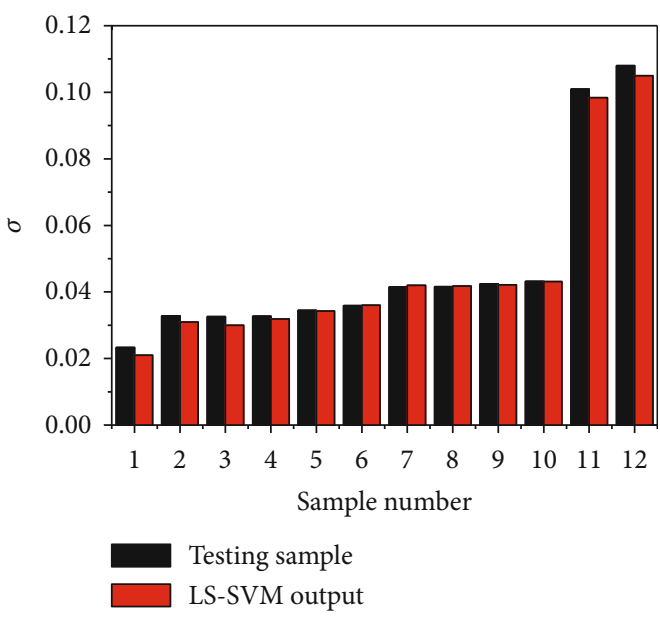

(b) Nonuniformity

FIgURE 7: Comparison of LS-SVM calculated value and testing samples.

Step 2. Establish the LS-SVM surrogate model based on the training samples and determine the empirical parameters in the model. The generation ability of the surrogate model is tested by the testing samples.

Step 3. Determine the optimal design parameters of the turbine chamber by the chaotic optimization algorithm.

In the present studies, three cases including singleobjective optimization for discharge coefficient, singleobjective optimization for outflow nonuniformity, and multiobjective optimization were performed.

\subsection{Numerical Model and Validation}

3.2.1. Numerical Simulation. Latin hypercube sampling (LHS) is an approximate random sampling method from the multivariate parameter distribution. It belongs to hierarchical sampling technology. Its advantage is that it can ensure the full coverage of each variable range. In the present study, $48(=12 \times \mathrm{n}$, where $\mathrm{n}$ is the dimension of designing

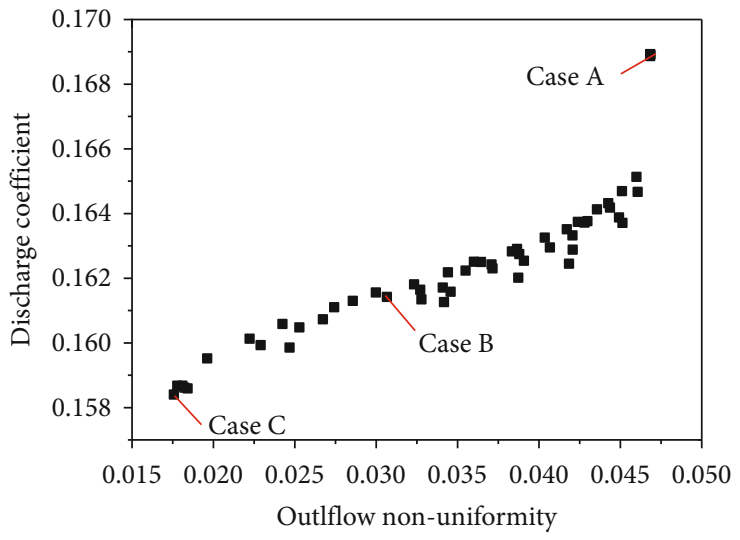

Figure 8: Pareto solution set. 
TABle 3: Optimized geometric parameters at different index weights.

\begin{tabular}{lcccccccc}
\hline & $\gamma$ & $\triangle(\mathrm{mm})$ & $q\left(^{\circ}\right)$ & $D(\mathrm{~mm})$ & $a\left(^{\circ}\right)$ & $C_{\boldsymbol{d}}$ & $\sigma$ & $\gamma \times \sigma+(1-\gamma) /\left(100 \times C_{d}\right)$ \\
\hline Case A & 1 & 10.2 & 76.9 & 46 & 0.9 & 0.171 & 0.0459 & 0.0459 \\
Case B & 0.5 & 17.9 & 89.0 & 45.3 & 3.8 & 0.163 & 0.0312 & 0.0463 \\
Case C & 0 & 9.9 & 80.3 & 41.3 & 6.1 & 0.156 & 0.0182 & 0.0641 \\
\hline
\end{tabular}

space) groups of data sample obtained through LHS were used for training samples, and 8-fold crossvalidation scheme was applied to determine the optimal empirical parameters in the surrogate model. 12 groups of testing samples generated randomly were used for testing samples.

ICEM software was used to generate the unstructured grids. The grids in the wall region are refined to ensure $y$ $+\approx 1.0$, and the stretch factor on the wall-normal direction is 1.1 1.2. Enhanced wall function was used for modeling near-wall flow. The grid number is determined by the grid independent test. Figure 4 shows the grid details and grid independent test results for the reference structure. The optimal grid number is about 5 million.

Gas governing equations were solved by Ansys Fluent software. Because the inlet Mach number is lower than 0.3, incompressible ideal gas was used in the present study. The chamber inlet was specified as pressure-inlet condition. The inlet total pressure and temperature are 1.2 $\mathrm{MPa}$ and $700 \mathrm{~K}$, respectively. The chamber outlet was specified as pressure-outlet condition, and the static pressure is 1.06 MPa. The walls were specified as adiabatic and nonslip condition.

The results from Yao et al.[6] show that the relizable $k-\varepsilon$ turbulent model can predict the flow behavior in the turbine chamber with high accuracy. In the present study, the relizable $k-\varepsilon$ model was also used. Second-order upwind scheme was used for solving momentum, turbulent kinetic energy, dissipation rate, and energy terms. The SIMPLEC algorithm is used for the coupling of pressure and velocity. The convergence criteria are that the residual error decreases to $10^{-6}$.

3.2.2. Model Validation. Figure 5 shows the experimental system which consists of gas supply system, gas heating system (not used in the present experiments), measurement system, and test section. The gas is supplied by a screw compressor with a rated volume flow of $37 \mathrm{~m}^{3} / \mathrm{min}$. The air mass flow rate is measured by a vortex flowmeter with the testing range of $5 \sim 50 \mathrm{~m}^{3} / \mathrm{min}$ and accuracy grade of 1.0 . After passing through the adapter, the air flow is divided into four channels, and the flow distributions are controlled by four valves to achieve the balance. The air flow enters the turbine chamber from four inlet tubes and finally leaves out from outlet hole. In the experiment, the hot bulb anemometer (st-732 model and $0.01 \mathrm{~m} / \mathrm{s}$ resolution) is used to measure the mean outflow velocity of each outlet hole. Accordingly, the mass flow from each outlet hole can be calculated. The inlet mass flow rate and temperature are $2.0 \mathrm{~kg} / \mathrm{s}$ and $300 \mathrm{~K}$, respectively. The outlet static pressure is $0.1 \mathrm{MPa}$.

The geometric parameters of the tested turbine chamber are the same to that in Table 1.

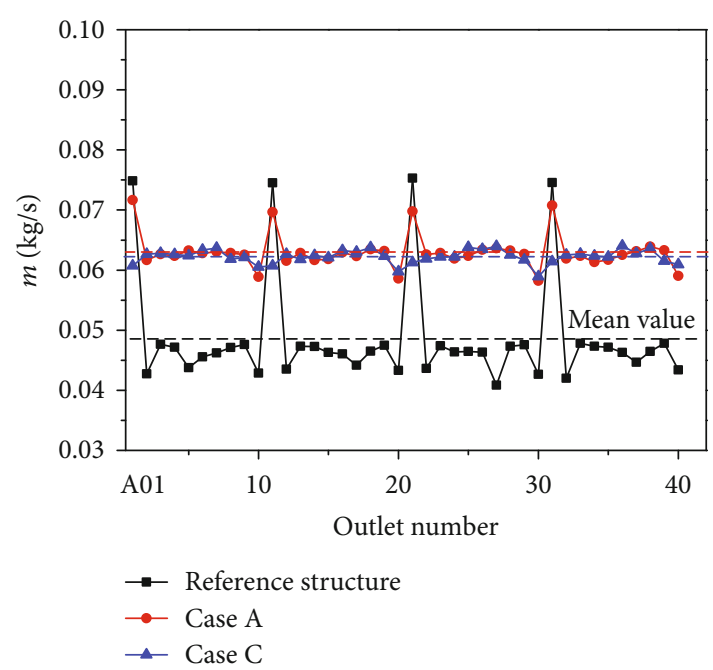

FIGURE 9: Outflow distribution for turbine chamber before and after optimization.

Figure 6 compares the outflow distribution profile in the CFD and experimental results. It should be noticed that outer annular chamber is not used in the experimental tests and CFD validation model. There exists four maximum points corresponding to the outlet holes facing the inlet tubes directly. This is due to the direct jet impingement of the gas. In the middle region between two adjacent inlet tubes, there exists a minimum outflow point. The averaged prediction error for nonuniformity is $3.1 \%$. Overall, CFD results agree well with the experimental results.

3.3. Surrogate Model. Given a series of data samples $G=\{($ $\left.\left.\boldsymbol{I}_{\mathrm{i}}, O_{\mathrm{i}}\right), i=1,2, \cdots, N\right\}\left(\boldsymbol{I}_{i}\right.$ and $O_{i}$ are the input vector and output value respectively, and $N$ is the size of data samples) to be fitted, based on the basic theory of LS-SVM, this regression problem can be modeled as $[11,12]$

$$
f\left(\mathrm{I}_{j}\right)=\sum_{i=1}^{N} O_{i} a_{i} \operatorname{ker}\left(\mathrm{I}_{j}, \mathrm{I}_{i}\right)+b,
$$

where $\operatorname{ker}(\cdot)$ is the kernel function, and $f\left(\mathbf{I}_{\mathbf{j}}\right)$ is the output value of LS-SVM. a and $b$ can be determined by solving the following equations:

$$
\left[\begin{array}{cc}
0 & \mathrm{~A}_{l} \\
\mathrm{~A}_{l}^{T} & \boldsymbol{\Omega}+\eta^{-1} \mathbf{E}
\end{array}\right]\left[\begin{array}{l}
b \\
\mathrm{a}
\end{array}\right]=\left[\begin{array}{l}
0 \\
\mathrm{O}
\end{array}\right],
$$

where $\eta$ is the penalty factor, $\boldsymbol{O}=\left[O_{1}, O_{2}, \cdots, O_{\mathrm{Ns}}\right]^{T}, \boldsymbol{A}_{1}$ 


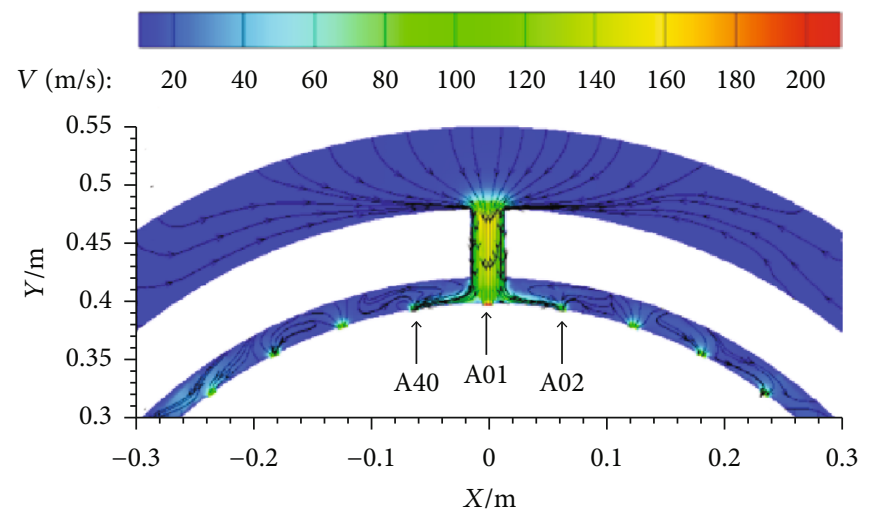

(a) Velocity distribution

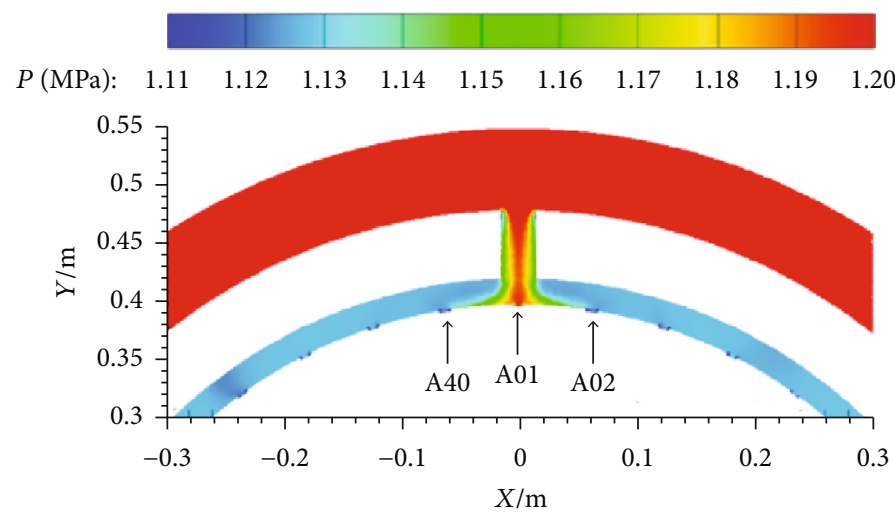

(b) Pressure distribution

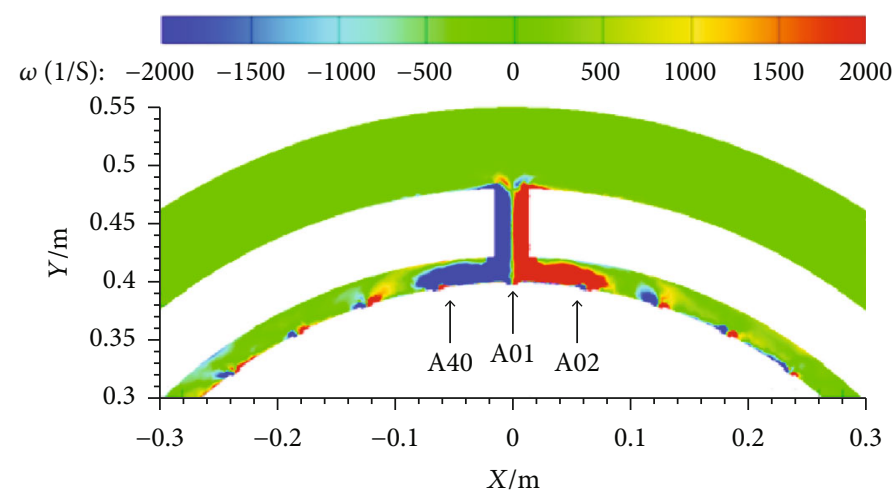

(c) Vorticity distribution

FIGURE 10: Velocity, pressure, and vorticity distributions inside the reference chamber.

$=[1,1, \cdots, 1], \boldsymbol{a}=\left[a_{1}, a_{2}, \cdots, a_{N}\right]^{T}, \boldsymbol{E}$ is the unit matrix, and $\boldsymbol{\Omega}(i, j)=k\left(I_{i}, I_{j}\right)$. In current research, the radial basis function is selected as the kernel function:

$$
\operatorname{ker}\left(\mathrm{I}_{i}, \mathrm{I}_{j}\right)=\exp \left(-\frac{\left\|\mathrm{I}_{i}-\mathrm{I}_{j}\right\|}{2 \delta^{2}}\right) \text {, }
$$

where $\delta$ is the kernel parameter.

The surrogate model is established by SVM toolbox in Matlab software. The prediction performance of LS-SVM depends on the values of penalty factor and kernel parameter. In the present study, the trial and error method and 8- fold crossvalidation scheme were used for determining these parameters. Figure 7 shows the comparison between the calculated values by LS-SVM and the testing samples (calculated by CFD). The averaged relative error for discharge coefficient is $2.3 \%$, and the averaged error for nonuniformity is $4.2 \%$.

3.4. Chaotic Optimization Algorithm. Different from traditional genetic or evolutionary algorithms that escape from the local optimal value by accepting some bad solutions according to a certain probability, the chaotic optimization algorithm searches on the regularity of the chaotic motion to escape from the local optimal value [13-15]. In the 


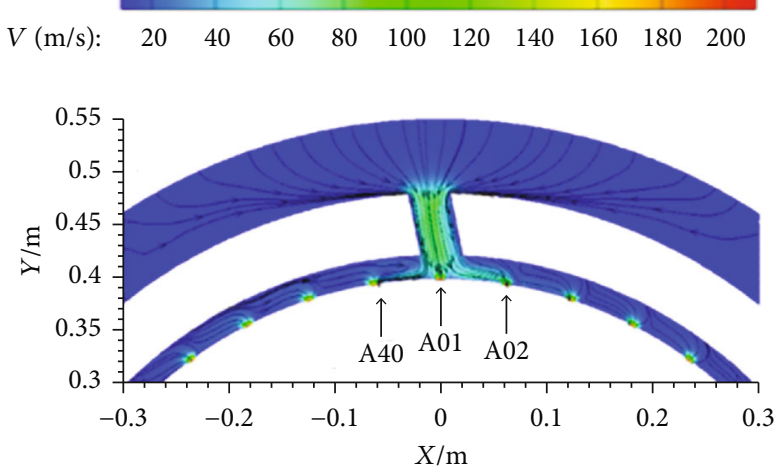

(a) Velocity distribution
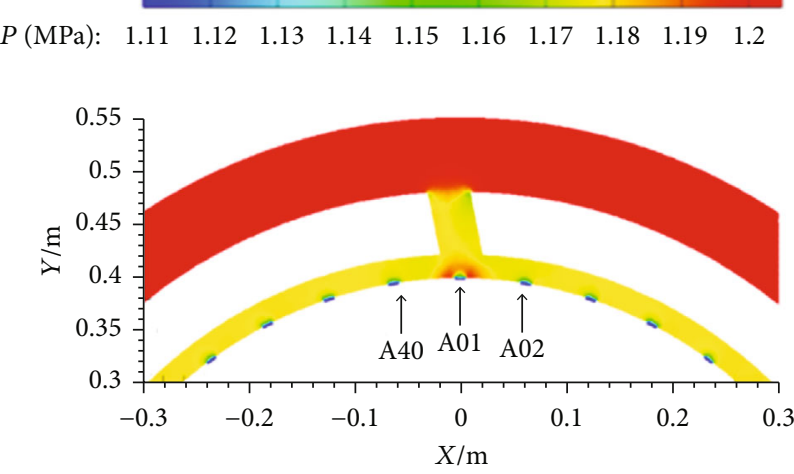

(b) Pressure distribution
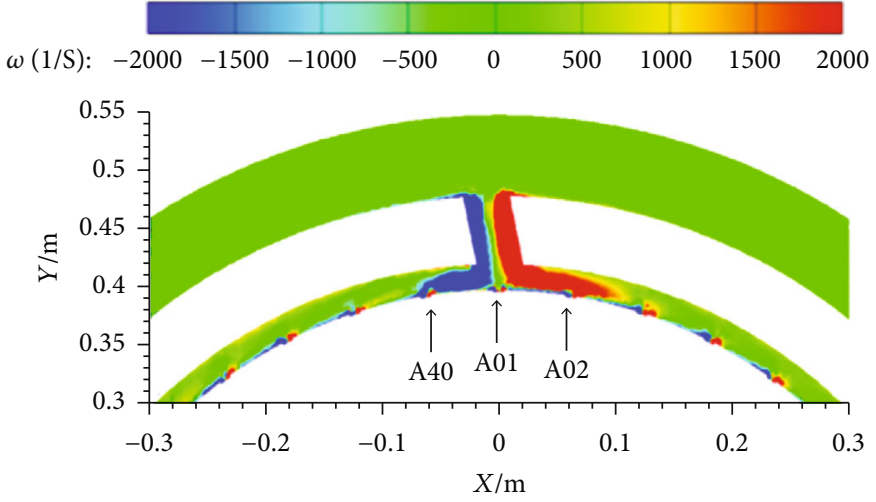

(c) Vorticity distribution

FIGURE 11: Velocity, pressure, and vorticity distributions inside the chamber of Case A.

present study, the logistic model is selected as chaotic model to generate chaotic time series by iteration. The expression of the logistic model is shown as follows:

$$
t_{m+1}=\lambda t_{m}\left(1-t_{m}\right) \lambda \in(0,4.0)
$$

The basic steps of the chaotic optimization algorithm can be divided into 9 steps [15]:

Step 1. Give $N_{1}$ and $N_{2}$ a large positive integer, respectively, where $N_{1}$ and $N_{2}$ is the maximum global and detailed search steps, respectively.

Step 2. Give $\boldsymbol{t}^{(1)}=\left(t_{1}{ }^{(1)}, t_{2}{ }^{(1)}, t_{3}{ }^{(1)}, t_{4}{ }^{(1)}\right)$ a 4-dimensional random vector and generate chaotic series $\boldsymbol{t}^{(j+1)}, j=1, \cdots$, $N_{1}$ by Eq. (9).

Step 3. Assign $\left(t_{1}{ }^{(n)}, t_{2}{ }^{(n)}, t_{3}{ }^{(n)}, t_{4}{ }^{(n)}\right)$ to normalized $\left(d_{\mathrm{in}}, \triangle\right.$, $\theta, \alpha)$ and calculate $F^{n}$ by LS-SVM. If $n$ equals 1 , then assign $F^{1}$ and $\mathbf{t}^{1}$ to $F^{*}$ and $\mathbf{t}^{*}$, respectively.

Step 4. If $F^{*}>F^{n}$, then assign $F^{n}$ and $\mathbf{t}^{(n)}$ to $F^{*}$ and $\mathbf{t}^{*}$.
Step 5. $n=n+1$. If $n$ is smaller than $N_{1}$, return to step 3. If $n$ is larger than $N_{1}$, then generate $\left(t_{i, \text { min }}^{\prime}, t_{i \text {,max }}\right)$ by

$$
\left\{\begin{array}{l}
t_{i, \min }^{\prime}=t_{i}^{*}-\varphi\left(t_{i, \max }-t_{i, \min }\right) \\
t_{i, \max }^{\prime}=t_{i}^{*}+\varphi\left(t_{i, \max }-t_{i, \min }\right)
\end{array}\right.
$$

where $\varphi$ is in the interval of 0 and 0.5 . If $t_{i, \min }^{\prime}$ is smaller than $t_{i, \min }$, then assign $t_{i, \min }$ to $t_{i, \min }^{\prime}$. If $t_{i, \max }$ is higher than $t_{i, \max }$, then assign $t_{i, \max }$ to $t_{i, \max }$.

Step 6. A new chaotic vector $\mathbf{t}^{m}$ can be generated by

$$
t_{i}^{m}=\left(1-\beta_{i}\right) \frac{t_{i}^{*}-t_{i, \text { min }}^{\prime}}{t_{i, \text { max }}^{\prime}-t_{i, \text { min }}^{\prime}}+\beta_{i} \frac{t_{i}^{m}-t_{i, \text { min }}}{t_{i, \text { max }}-t_{i, \text { min }}},
$$

where adaptive adjustment coefficient, $\beta_{i}$, can be determined by the following equation:

$$
\beta_{i}=1-\left(\frac{m-1}{m}\right)^{l}
$$

where $l$ equals 2 in the present study. 


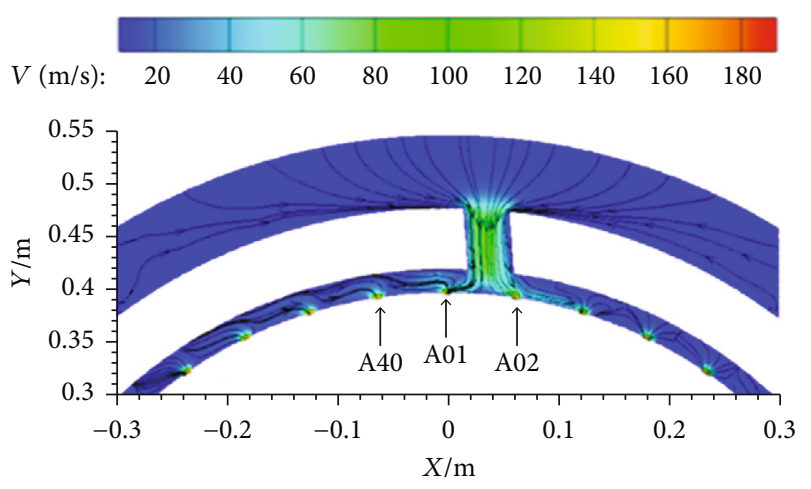

(a) Velocity distribution

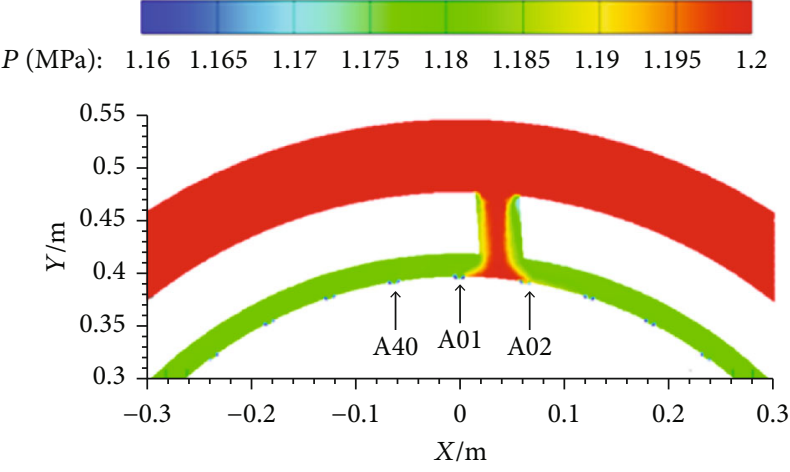

(b) Pressure distribution

$$
\omega(1 / \mathrm{S}):-2000-1500-1000-500 \quad 0 \quad 500 \quad 1000 \quad 1500 \quad 2000
$$

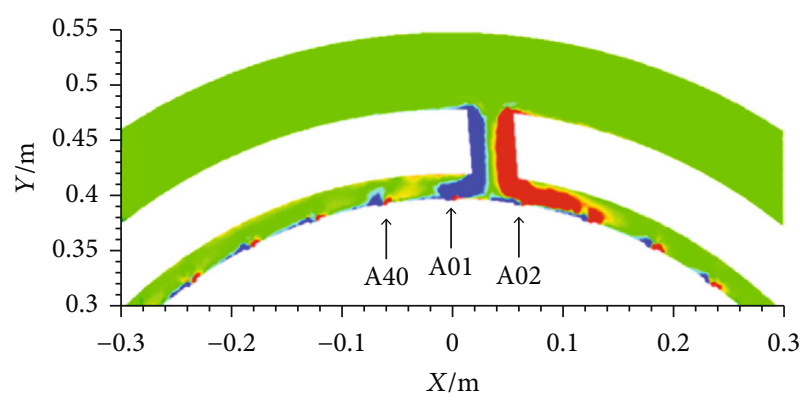

(c) Vorticity distribution

FIgURE 12: Velocity, pressure, and vorticity distributions inside the chamber of Case C.

Step 7. Give $\left(t_{1}{ }^{(m)}, t_{2}{ }^{(m)}, t_{3}{ }^{(m)}, t_{4}{ }^{(m)}\right)$ to normalized $\left(d_{\mathrm{in}}, \triangle\right.$, $\theta, \alpha)$ and calculate $F^{n}$. If $m$ equals to 1 , then assign $F^{1}$ and $\mathbf{t}^{1}$ to $F^{*}$ and $\mathbf{t}^{*}$ respectively.

Step 8. If $F^{*}$ is larger than $F^{n}$, assign $F^{n}$ and $\mathbf{t}^{(m)}$ to $F^{*}$ and $\mathbf{t}^{*}$

Step 9. $m=m+1$. If $m$ is smaller than $N_{2}$, then go to the 6 th step. If $m$ equals to $N_{2}$, the optimization processes end.

The chaotic optimization is performed by inhoused codes. Set the maximum global search step $N_{1}=4000$ and the maximum detailed search step $N_{2}=2000$. Figure 8 shows the distribution of Pareto solution set for multiobjective optimization. 60 optimization processes with different index weights were performed, and each point in Figure 8 corresponds to an index weight. For example, case A corresponds to the weight of 0 , and the optimization goal is maximizing discharge coefficient. Case B corresponds to the weight of 1.0, and the optimization goal is minimizing outflow nonuniformity. Case $\mathrm{C}$ corresponds to the weight of 0.5 , and the discharge coefficient and outflow nonuniformity were taken into consideration simultaneously. The detailed optimized geometric parameters are shown in Table 3.

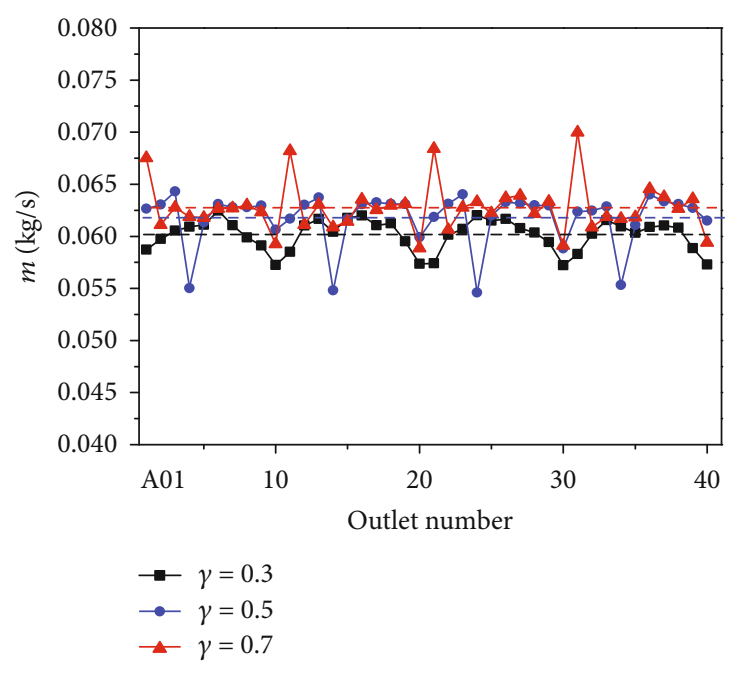

FIGURE 13: Outflow distribution after multiobjective optimization.

\section{Results Analysis}

4.1. Single-Objective Optimization. Figure 9 compares the outflow distribution characteristics before and after optimization. As listed in Table 3, case A corresponds to the optimized structure for discharge coefficient. Case C corresponds to the optimized structure for outflow uniformity. Compared with 


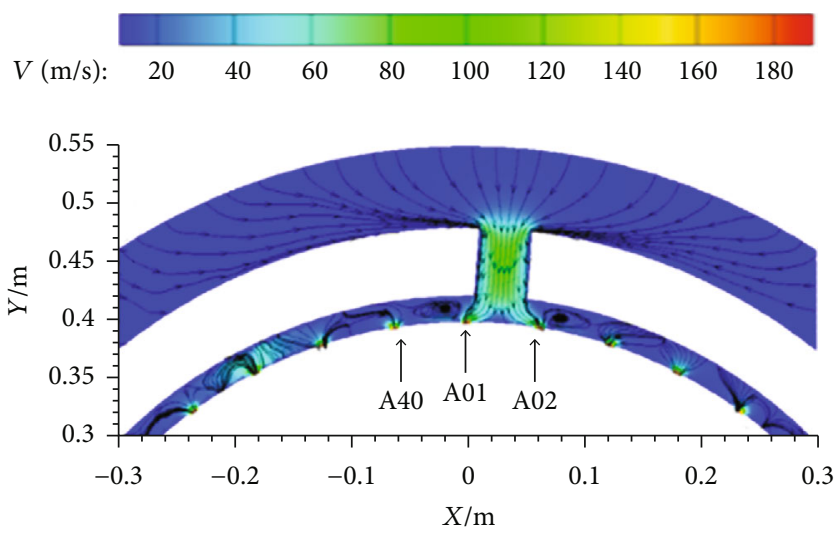

(a) Velocity distribution

$\begin{array}{llllllllllll}P(\mathrm{MPa}): & 1.15 & 1.155 & 1.16 & 1.165 & 1.17 & 1.175 & 1.18 & 1.185 & 1.19 & 1.195 & 1.2\end{array}$

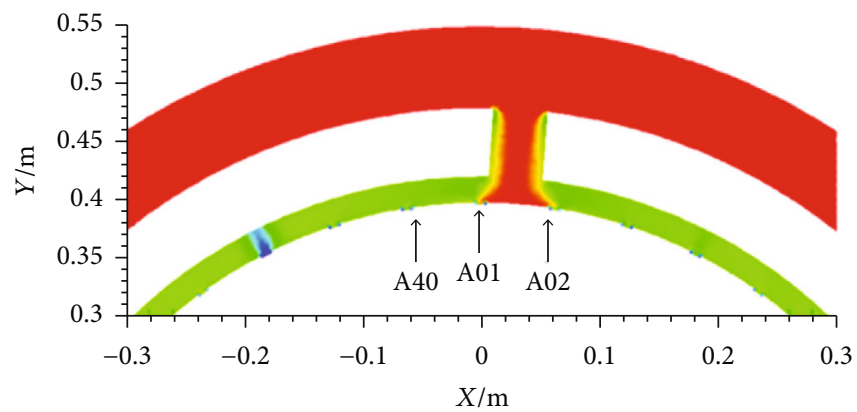

(b) Pressure distribution
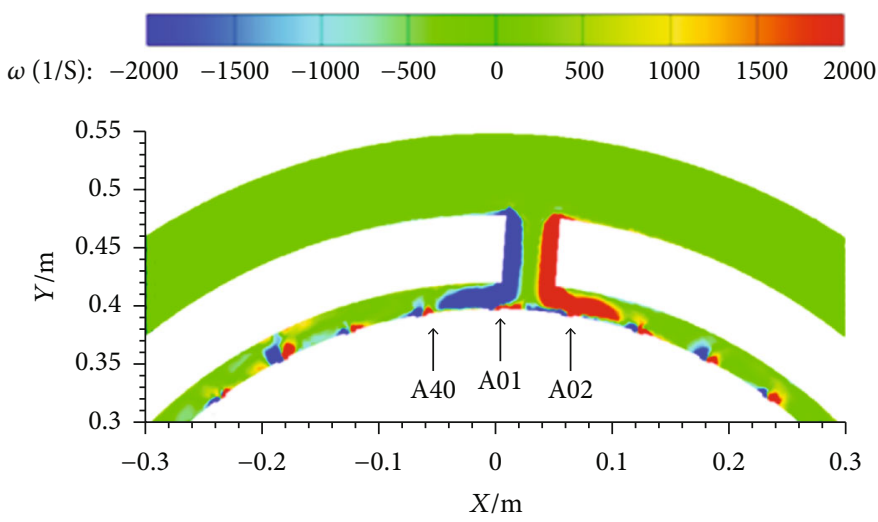

(c) Vorticity distribution

Figure 14: Velocity, pressure, and vorticity distributions in the optimized chamber with $\gamma=0.5$ (case B).

the reference structure, the mass flow rate for case A increases by $30 \%$ at the same pressure ratio, while the nonuniformity of outflow decreases by $76 \%$. For the outflow distribution profile of case $\mathrm{A}$, there exists four maximum points. The maximum value of outflow distribution profile decreases, but the minimum value increases obviously after optimization. For case $\mathrm{C}$, the mass flow rate increases by $26 \%$, and the nonuniformity of outflow decreases by about $90 \%$. Moreover, compared with the reference structure and case A, the outflow distribution profile of case $\mathrm{C}$ is more uniform. Overall, by optimization, the flow resistance is reduced, while the outflow uniformity is improved effectively.
Figure 10 shows the velocity, pressure, and axial vorticity distribution inside the reference chamber. A01 faces the inlet tube, and the coolant from the inlet tube enters A01 directly. Accordingly, in the inlet region of A01, the flow pressure is fully utilized, and the high-speed air flow does not impinge with the chamber wall directly. On both sides of A01, the dynamic pressure converts to static pressure which forms an obvious high-pressure belt, and the coolant flow changes from the wall-vertical direction to the wall-parallel direction. A02 and A40 locate in the wall-flow region. The coolant entering A02 and A40 is much smaller than A01. In the 
middle area between the two inlet tubes, due to high energy loss and low total pressure, the mass flow rate is also very low.

Figure 11 shows the velocity, pressure, and vorticity distributions inside the chamber of case A. Case A corresponds to the optimized structure for discharge coefficient. The inlet diameter of inlet tube is $46 \mathrm{~mm}$, and the inclination angle is $76.9^{\circ}$. At the same pressure ratio, adding the inlet duct diameter can results in the increase of mass flow rate. From Figure 11(a), it can be seen that the inclination of the inlet tube can increase the tangential velocity component of injection air and reduce the mass flow rate from A01. Accordingly, the outflow from A01 in case A is less than that in the reference structure. Moreover, because of the inclination injection, the outflow from the holes on both sides of A01 increases compared with the reference structure. Besides of these advantages, the inclined inlet can also reduce the flow loss inside the chamber.

Case C corresponds to the optimized structure for outflow uniformity. As shown in Figure 12, due to the effect of included angle between inlet and outlet $(\alpha)$, there is no outlet hole at the position facing the inlet tube directly, which avoids the maximum outflow value. The coolant from the inlet impinges with the inner wall of the chamber at a high speed, which results in formation of an obvious highpressure zone in the wall region. Under the effect of pressure gradient, the gas flows along the chamber wall and then leaves the chamber from the outlet hole. The outflow from the outlet hole gradually decreases along the flow direction. In the middle area of the two inlet tubes, the coolant from contrary directions collides with each other and leaves from the outlet hole. Therefore, there is no obvious maximum and minimum of outflow in the structure of case $C$, and the outflow distribute profile is uniform. Compared with the reference structure, the outflow nonuniformity decreases about $90 \%$.

4.2. Multiobjective Optimization. Figure 13 shows the optimized outflow distribution profiles with different index weights. As $\gamma=0.3$, the mean mass flow rate for each hole is $0.0573 \mathrm{~kg} / \mathrm{s}$, and the nonuniformity is 0.0244 . As $\gamma=0.5$, the mean mass flow rate for each hole is $0.0615 \mathrm{~kg} / \mathrm{s}$, and the nonuniformity is 0.0312 . As $\gamma=0.7$, the mean mass flow rate for each hole is $0.0594 \mathrm{~kg} / \mathrm{s}$, and the nonuniformity is 0.0443 . Take the optimization result with $\gamma=0.5$ as example, Figure 14 shows the distributions of velocity, pressure, and vorticity inside the chamber. After optimization, the diameter of the inlet tube is $45.3 \mathrm{~mm}$, and the circumferential deflection angle is $3.8^{\circ}$. Compared with reference structure, the discharge coefficient shows obvious increase, and the outflow distribution is more uniform because that there is no outlet facing the inlet tube directly. Moreover, the coolant jets directly impact with the chamber wall and result in the formation of complex vortices around A02 and A40. Under the effect of pressure gradient, the coolant flows along the chamber wall and leaves the outlet hole. After optimization, the axial deviation is $17.9 \mathrm{~mm}$. The existence of axial deviation can also reduce the direct jet effect of coolant to the outlet and improves the outflow uniformity. Moreover, due to the slight inclination of the inlet tube, only a small amount of coolant can reach the outlet in the middle of the two inlet tubes; accordingly, the pressure in this region is low.

\section{Conclusions}

(1) An optimization method by coupling LS-SVM and chaotic optimization algorithm was developed for turbine coolant collecting/distribution chamber. Firstly, the numerical experiments were designed by the Latin hypercube sampling method, and the data samples are obtained by CFD solution; the surrogate model of LS-SVM is established, and the parameters of the surrogate models are determined based on the training samples; Finally, a kind of chaotic optimization algorithms is introduced to search for the optimization variables globally.

(2) The single objective optimization is carried out. As the discharge coefficient is taken as the optimization objective, the optimal structure has axial deviation of $10.2 \mathrm{~mm}$, inlet duct inclination angle of $76.9^{\circ}$, inlet duct diameter of $46 \mathrm{~mm}$, and included angle between inlet and outlet of $0.9^{\circ}$. As the outflow nonuniformity is taken as the optimization objective, the optimal structural has an axial deviation of $9.9 \mathrm{~mm}$, inlet duct inclination angle of $80.3^{\circ}$, inlet duct diameter of $41.3 \mathrm{~mm}$, and included angle between inlet and outlet of $6.1^{\circ}$.

(3) The multiobjective optimization of turbine chamber structure was carried out by considering discharge coefficient and outflow nonuniformity simultaneously. The optimized geometric parameters of turbine chamber under different weight conditions were determined, and the flow characteristics were analyzed in detail.

In the present study, only outflow nonuniformity is considered. In fact, the nonuniformity of outlet temperature also affects the cooling efficiency of turbine nozzles. The optimization considering the effect of outlet temperature nonuniformity is worthy of further study.

\section{Nomenclature}

$C_{d}: \quad$ Discharge coefficient (-)

$d_{\text {in }}$ : Diameter of inlet tube $(\mathrm{mm})$

$d_{\text {out }}$ : Diameter of outlet hole $(\mathrm{mm})$

$H$ : Height of turbine coolant chamber ( $\mathrm{mm}$ )

$m$ : Mass flow rate $(\mathrm{kg} / \mathrm{s})$

$N_{\text {in }}$ : Number of inlet tube (-)

$N_{\text {out }}$ : Number of outlet hole (-)

$P_{\text {in }}^{*}:$ Inlet total pressure $(\mathrm{Pa})$

$P_{\text {out }}:$ Outlet static pressure $(\mathrm{Pa})$

$R_{1}$ : Inner radius at the front end of turbine coolant chamber $(\mathrm{mm})$

$R_{2}$ : Inner radius at the trailing end of turbine coolant chamber (mm)

S: $\quad$ Axial length of turbine coolant chamber ( $\mathrm{mm})$ 
$V: \quad$ Gas velocity $(\mathrm{m} / \mathrm{s})$

\section{Greek Symbols}

$\Delta:$ Axial space between inlet and outlet $(\mathrm{mm})$

$\theta$ : Inclination angle of inlet tube $\left(^{\circ}\right)$

$\alpha$ : Included angle between inlet and outlet $\left({ }^{\circ}\right)$

$\sigma$ : Outflow nonuniformity (-)

$\gamma$ : Index weight (-)

$\delta$ : Kernel parameter in LS-SVM (-)

$\omega$ : Axial vorticity $\left(1 / s,=\left(\partial V_{\mathrm{x}} / \partial y\right)-\left(\partial V_{\mathrm{y}} / \partial x\right)\right)$.

\section{Data Availability}

The data that support the findings of this study are available from the corresponding author upon request

\section{Conflicts of Interest}

The authors declare that there is no conflict of interest regarding the publication of this paper.

\section{Acknowledgments}

This study was supported by the National Science and Technology Major Project of China (grant No: 2017-III-00110037).

\section{References}

[1] A. H. Nissan and V. P. Bresan, "Swirling flow in cylinders," AICHE Journal, vol. 7, no. 4, pp. 543-547, 1961.

[2] S. Kumar and R. S. Amano, "Experimental investigation of heat transfer and flow using $\mathrm{V}$ and broken $\mathrm{V}$ ribs within gas turbine blade cooling passage," Heat and Mass Transfer, vol. 51, no. 5, pp. 631-647, 2015.

[3] S. W. Lee, H. S. Moon, and S. E. Lee, “Tip gap height effects on flow structure and heat/mass transfer over plane tip of a highturning turbine rotor blade," International Journal of Heat and Fluid Flow, vol. 30, no. 2, pp. 198-210, 2009.

[4] R. S. Bunker and D. E. Metzger, "Local heat transfer in internally cooled turbine airfoil leading edge regions: part Iimpingement cooling without film coolant extraction," ASME Journal of Turbomachinery, vol. 112, no. 3, pp. 451-458, 1990.

[5] S. Zhao, "Experimental research on flow characteristics of turbine gas collecting chamber," in The 7th Power Annual Conference of Chinese Aeronautical Society, Guiyang, China, 2010.

[6] T. Yao, J. K. Mao, and L. L. Jiang, "Research on the flow characteristics of turbine gas collecting chamber and the calculation model of flow resistance," Journal of Propulsion Technology, vol. 38, pp. 364-375, 2017.

[7] X. Y. Zhang, An investigation on massflow distribution in a turbine blade Coolant-Collecting plenum, Master thesis, Nanjing University of Aeronautics and Astronautics, 2014.

[8] K. D. LEE, "Multi-objective optimization of a laidback fan shaped film-cooling hole using evolutionary algorithm," International Journal of Fluid Machinery and Systems, vol. 3, no. 2, pp. 150-159, 2010.

[9] Y. Huang, "Optimization of fan-shaped holes on turbine blade suction surface to improve film cooling performance," Journal of Central South University, vol. 49, pp. 2868-2876, 2018.
[10] C. Wang, J. Z. Zhang, and C. H. Wang, "Multi-optimization of a specific laminated cooling structure for overall cooling effectiveness and pressure drop," Numerical Heat Transfer Part A: Applications, vol. 79, pp. 1-27, 2020.

[11] C. Cortes and V. Vapnik, "Support-vector networks," Machine Learning, vol. 20, no. 3, pp. 273-297, 1995.

[12] C. H. Wang, J. Z. Zhang, and J. H. Zhou, "Prediction of filmcooling effectiveness based on support vector machine," Applied Thermal Engineering, vol. 83, pp. 82-93, 2015.

[13] H. Shayeghi, H. A. Shayanfar, S. Jalizadeh, and A. Safari, "Multi-machine power system stabilizers design using chaotic optimization algorithm," Energy Conversion and Management, vol. 51, no. 7, pp. 1572-1580, 2010.

[14] C. L. Santos, "Tuning of PID controller for an automatic regulator voltage system using chaotic optimization approach," Chaos Solitons Fractals, vol. 39, no. 4, pp. 1504-1514, 2009.

[15] Y. Z. Zhao and C. H. Wang, "Shape optimization of labyrinth seals to improve sealing performance," Aerospace, vol. 8, no. 4, p. $92,2021$. 